\title{
THE RUMORS SYSTEM OF RUSSIAN SYNTHESIS
}

\author{
Max I. Kanovich, Zoya M. Shalyapina \\ Institute of Oriental Studies, Russian Academy of Sciences, \\ Rozhdestvenka str., 12, 103753 Moscow, Rnssia
}

\begin{abstract}
The RUMORS synthesizer of Russian is an integral part of the JaRAP experimental system of JapaneseRussian automatic translation, allhough if cun ulso have other applications. Morphologically, it is based, primarily, on A.A.Zuliznyak's model of Rusaian inflexion. Syntactical functions of RUMORS rely on word-order and dependency data as input information. The synthesizer is implemented on IIIM PC, MS DOS, in Turbo Puscul.
\end{abstract}

\section{General information}

The RUMOIR system of RUsaian MORphological and Syntactical synthesis has been developed as part of the JaRAP experimental system of $J$ apanescRussian antomatic tranglation, described in (Modina, Shalyapina, 1094). Ito operation is, however, completely independent of the other components of the JaRAP aystem, so that RUMORS could be used in any other AT system irrespective of its gource language. Basically, RUMORS constitutes a system in its own right, which can also be naed for purposes other than translation, e.g., as a computerized reference book of Russian morphology and the simplest phenomena of syntactic government and agrcement (for students and teachens of Russian), us part of a spell-checking system, etc.

The RUMONS synthesizer has two major modes of operation: the QUERY mode and the TASK mode.

In the QUCHY mode of operation, HUMOIS accepts, as ita input, a separate syntacticomorphological query (entered from the keybonrd) which represents the lexeme to be processed and the syntactical and morphological characteristics specifying the word-form to be obtained by the processing. The output in, primarily, the degired word-form of the input lexeme or, if necessary, a periphrastic substitute for this word-form.

After obtaining this output, the user cas switch at will to the FULL PARADIGM submode of the QUERY mode. In this unbmode, RUMORS generates all synthetic word-forms of the input lexeme (or of the last lexeme processed while obtaining a pe- riphrastic word-combination). It also offers a set of menus allowing the user to modify his initial anery by choosing additional morphological categories [ron these menus.

In the 'I'ASK mode, the inpul data is a sequence of queries fed from the special TASK file. Apart from lexicul, morphological, and syntactical informar tion contained in each query, the TASK mode of operation makes considerable nse of word order data which is essentisal, among other things, for processing prepositional, adjectival and noun plurases. 'The output is the sequence of word-forms manifesting the phrases or sentences specified by the input sequence of querie日.

Both in the QUERY und in the TASK modes, the output is displayed on the screen and written simnltaneously in the special SOLVE file. If required by the user, it may also include the alterations made in the queries processed and the database information used in their processing. Inasmuch us the simulation by RUMORS of the linguiatic processes involved in Mussian synthesis is faithful enough, this auxiliary datr could be vuluable by itself (e.g., for learning or teaching Russian), aside from its significance for debugging und controlling purposes.

\section{Synthesis functions envisaged by RUMORS}

\subsection{Morphological functions}

The morphological functions of RUMOILs cover all agpects of Russian inflexion, as well as some semantically banic lexico-morphological relutionships.

'The inflexional functions are initiuted after the input query has already been subjected to syntactical and lexico-morphological operations, which muy have modified its initial form. At this stage, it contains nothing but the lexeme and the inflexionul categories specifying its desired word-form. If some cutegories needed for complete apecification of this word-form are not explicitly stated in the query, they are settled by default. E.g., a query containing nothing but the lexeme of a verb is taken to describe the finite 
form, indicative mood, present tense, active voice, $3 \mathrm{~d}$ person singular of this verb, so that the query, say, 6 a ams produces the form 6 epem.

The inflexional model of Rasaian implemented by RUMORS is the one proposed and detailed by prof. A.A.Zaliznyak (1977). Ito important virtue is that the generation procedures it envisages represent those to be expected of human aperkers of Russian more faithfully than any other known model, while the requisite database information is very compact.

Our version of 'Zaliznyak's inflexional model differs from its description in (Zaliznyak, 1977) in two respects. On the one hand, we have reduced the scope of the original Zaliznyak's model, implementing it only in so far as written Russian is concerned. As a result, quite a number of the particulars of Russian accentuation registered in (Zaliznyak,1977), namely, all those that are relevant for oral apeech only, have been ignored.

On the other hand, we have extended the model to cover analytical word-formb. Moreover, we have introduced a new type of morphological fanctions, the periphrastic functions allowing RUMORS to produce output that makes sense even if the required wordform is non-existent (e.g., due to the lexeme having a defective paradigm or to the combination of categories in the query being beyond the scope of Russian inflexional morphology). E.g., the future tense 1st person singular of the verb

no6edums 'win'

(which does not have this form) is parephrased as

csoay nobedumb ' $<I>$ shall be able to win'.

The lexico-morpholagical functions of $\mathrm{RU}$ MORS are limited so far to conversion between lexemes having essentially similar semantics, but differing in their partwof-speech or (for verbs only) aspectual characteristics. 'Thus, the aspectual or part-ofspeech markers in the following three querieg

\section{pasocmsamb:cos, พumamb:IIL, sкamb:II}

cause the lexemes in these queries to be replaced, resp., by the required perfective verb, noun, and ad jective:

\section{paccmusams, \\ rmerue, \\ usoecmsati".}

The implementation of aspectual

lexico-morphological relations is based, principally, on their description in (Zaliznya.k,1977). For purtof-apeech relations, we have adopted, though in a very limited sense, the concept of lexical substitutions (Zholkovskij,Mel'chuk, 1970).

If the databage contain no informution necessary for switching to a lexeme of the desired aspect or part of apeech, RUMORS resorts to its periphrastic functions or else makes modifications in the query.
E.g., the query

vuroesux: $I$

aimed a, forming the verb corresponding to the noun vนพовниx 'bureaucrat', will be processed to produce the phrase:

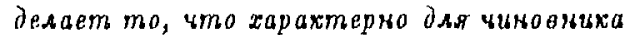

' $\langle I Y$ e $\rangle$ is doing what is typical of a bureaucrat'.

\subsection{Syntactical functions}

RUMORS has two major types of ayntactical functions: relational and word-atring ones. There is also a third group of prepositional functions.

Relational functions may be called both in the QUEHY and in the 'IASK mode of operation to modify the input query with regard to the relational references it may include. 'There may be references to dependency relations, where the node specified by the query acts either as dependent (D-references) or as governor ( $G$-references), and to anaphoric relations (F-references). D- and G-references may contain embedded relutionul references, so that in the general case each reference present in the input query corresponds to a more or less complex frugment of the dependency and anaphoric structure this query iв part of.

The job of the relational functions is to ensure fulfilment of the requirements for syntactical government and agreement which may be imposed on the word-form apecified by the query by the dependency and anaphoric relations this query has references to. This involves extracting such requirements from the references in the query, reconciling them with ench other (if there are two or more references dictating conflicting requirements), and then modifying the initial query to fit them: choosing the correct preposition or conjunction (the empty one, if needs be) to accompany the goal word-form, and altering, as required, the inflexional and part-of-speech categories within the query. E.g., the query

petuenue $n$ I)2(sasucems)

describing the noun peucsue 'decision' as the syntactical object of the verb saoucemb 'depend' will produce the prepositional combination:

om peuserus ' $<$ depend > on < the > decision'.

Word-string functions are specific to the TASK mode of operation only. Their peculiarity is that they include some analysis-like operations making it possible to locate and process aimple prepositional, uxljectival and noun phrases, even if the input sequence of queries has no syntactical marking.

To be more particular, word-string processing consiata in examining the queries of the input sequence one by one until the query under examination is found to answer our defirition of the end of is word-string. During this examination, each query is checked for informution relevant to agreement und preponitional government, and the inflexional and 
part-of-speech categories pertaining to snch informition are integrated into a special word-string query (w-query). After the end of the word-string has been located and examined, the w-query obtained is, in standard cases, made common to all of the individuat queries within this word-string. 'Thus, the sequence of morphologically empty queries for lexemes

o, oecs, saub, aasarmuxa 'in, all, our, galaxy'

will be processed to produce the preposition al phrase:

oo aceil rauell zasakmuxe

'in the whole of our galasy'.

Some types of word-strings, e.g. those containing cardinal numerals, have to be aubjected to more elaborate operations.

If a query within a word-string contains relational references, the requirements imposed by these are given priority over the requirements extracted by word-gtring functiona, so that the latter provide a sort of default.

Prepositional functions are employed in both modes of operation, if the word-form or word-string being processed is to be preceded by a preposition. Thus, if the preposition in question denotes location, direction or source, the noun it is meant to accompany is checked for having lexical preferences in thi respect. This helps to account, e.g., for guch idiomatica as

roe yause 'in the street'

v8.

n nepeyse 'in the side-street'.

Other prepositional functions serve to add the prothetic $\mathrm{N}$ to personal pronouns after prepositions imposing this requirement, to choose the contextual form of the preposition if it hat more thun one of them, etc.

\section{Database}

The database used by HUMORS has been derived, primarily, from (Luliznyak, 1977) which provider information on inflexion and uspectual conversion for about 100000 lexerner. We used a computerized ver. sion of (Zaliznyak, 1977) made avajlable to wa by the Department of the machine pool of the Russian language (Institute of the Rusaian language of the Russian rcademy of aciences, Moscow). By now, however, our database is appreciably different from its source.

Aside from various minor modifications, we have taken advantage of the fact that it is not unusual for inflexional information charactering a lcxeme to correlate with some componenty of this lexeme's word-atructure. Such components have been orgar nized into a dictionary of their own, the information associated with each of them included in their respective entries, and ull the corrresponding lexemes removed from the database.
The databuse has thus been reduced to less than one fifth of ('Zisliznyak,1977), ntill affording correct morphological processing of all of the 100000 lexemes listed in (Kadiznyak, 1977).

Moreover, so far as lexernes with standurd morphoJogical charucteristicn go, they can now be processed correctly, cven if they are newly-coined or occusionul (and do not have therefore dictionary entries of their own). As (Zaliznyak,1977) may be trusted to contrin all non-standard lexemes, the inflexional and aspectual information in the resulting database very nearly covers the whole of the Rusisian vocabulary.

The situation is different with information to be used in part-of-speech conversion and syntactic processing, for it is not provided in (Zaliznyak, 1977). 'This information is now also being added, but in this respect, the database is far from completed and has us yet only experimental value.

\section{References}

[1] L.S.Mollina, L.S., Shalyapinu, K.M. (1994). The $J_{a} H \Lambda \mathrm{P}^{3}$ experimental system of JoparieseRuвніนr untormatic translation (ambmitted for COLING 94).

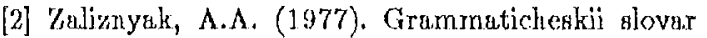
rustkogro yazyka (The G rammatical Dictionary of

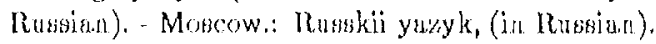

[3] Zholkovskij, A.K., Mel'chuk, 1.A. (1970). Sur la. synthèe sémantique. T.A.Information, No.2. 